\title{
La práctica educativa del licenciado en
} educación física

Proyecto de investigación

NeLLY TERESA MaRTiNeZ*

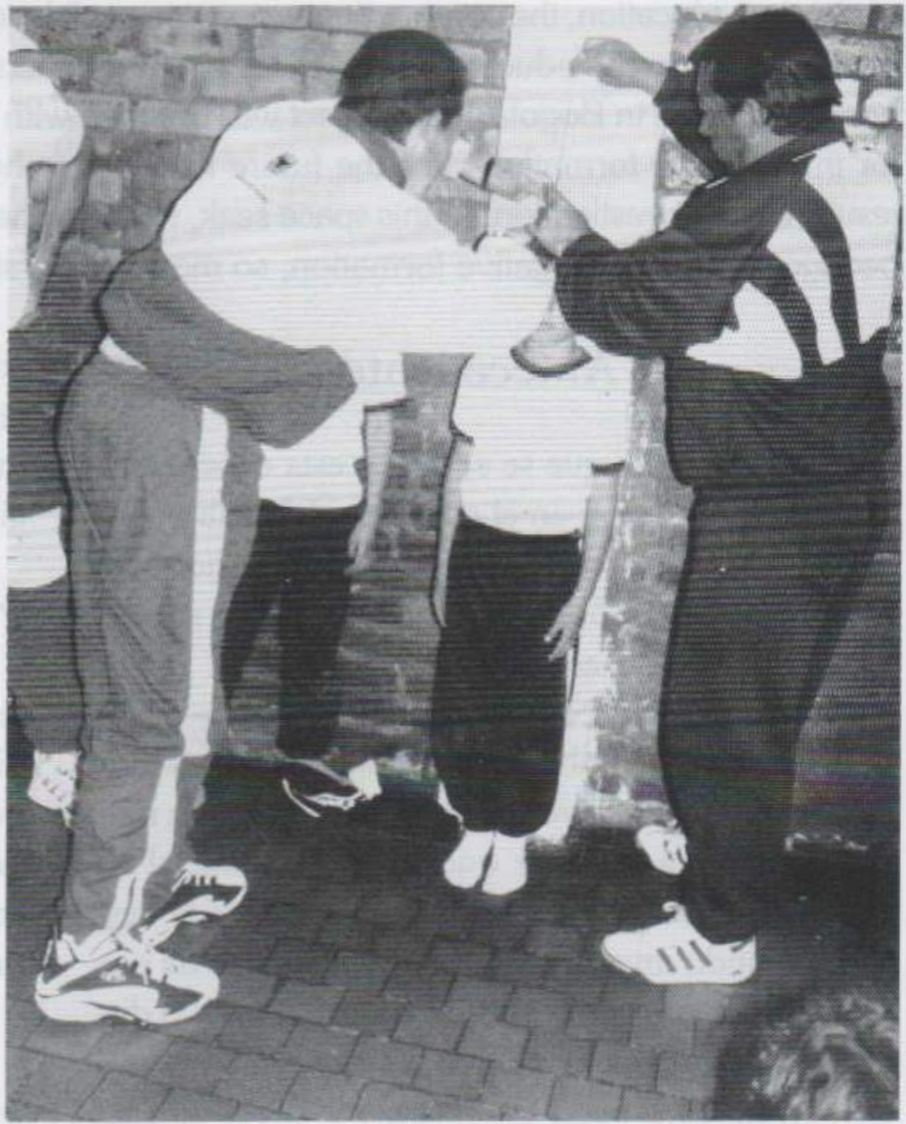

Por: Ricardo Neme Barrero

\section{Resumen}

9

undamentalmente, este artículo busca dar a conocer a la comunidad académica de formadores de maestros en el área de educación física, el proceso de construcción, consolidación y desarrollo inicial de un proyecto de investigación formativa sobre las prácticas educativas de los maestros que laboran en esta área, a nivel de la básica secundaria en Bogotá. El proyecto fue planteado con la intencionalidad de constituirse en un espacio para la formación reflexiva - crítica del futuro maestro de educación física; por este motivo, más que resultados finales de la investigación, en este espacio se pretende, dar a conocer el estado en que se encuentra y lo que ha aportado a la formación pedagógica e investigativa, tanto de los estudiantes como de los profesores vinculados a él.

Fecha de recepción: agosto 12 de 2003 - Fecha de aceptación: octubre 6 de 2003

* Profesora Facultad de Educación Física. Universidad Pedagógica Nacional. 
Palabras clave: práctica educativa, clase de educación física, educación, educación física, pensamiento técnico, pensamiento práctico.

\begin{abstract}
Fundamentally this article looks for to know to the academic community of teaching's teachers in the area of physical education, the construction process, consolidation and initial development of a project of formative investigation on the educational practices of the teachers that work in this area, at secondary level of the basic education in Bogotá. The project was outlined with the premeditation of being constituted in a space for the reflexive formation-critic the future teacher of physical education, for this reason, more than final results of the investigation, in this space seek, to know the state in that it is and what has contributed to the pedagogic and investigative formation, so many of the students as professors link to the one.
\end{abstract}

\section{Antecedentes}

El proyecto en que se enmarca esta experiencia de formación, surge en el contexto del análisis critico sobre los procesos de formación de licenciados en educación física y de la observación analítica de las prácticas educativas profesionales de los egresados de la UPN (Universidad Pedagógica Nacional). El largo trabajo ${ }^{2}$ de orientar los procesos de enseñanza de la investigación condujo, en un momento dado, a plantear que la práctica inicial de maestros , debería estar articulada y orientada desde un sentido investigativo, bajo la hipótesis de que ello contribuye a la formación de una actitud o disposición permanente que le induce a observar y cuestionar las propias prácticas, y a hacer de ellas un espacio que permita, al educador, develar, realmente, en su acción diaria, los pensamientos y creencias acerca del ser humano; a partir de los cuales orientaría su quehacer como maestro. Así, pensamientos y creencias generados desde los contextos sociopolíticos, culturales y pedagógicos de la formación y de su experiencia profesional como maestro, podría suscitar una resignificación del saber y una adaptación creativa a los contextos cambiantes.

Como consecuencia de este análisis, surgió la necesidad de plantear un proyecto de investigación que pensara la formación del estudiante y del maestro, en una doble dimensión: una investigativa y otra, pedagógica. Con la primera, se buscaría la adquisición de herramientas metodológicas propias de la investigación científica aplicada a la educación, y con la segunda, crear una mentalidad de estudio reflexivo y permanente de la práctica educativa, que impulse la construcción de nuevos saberes pedagógicos y didácticos.

\subsection{Surgimiento del proyecto}

$\mathrm{Al}$ iniciar el segundo semestre del 2001, se presenta a la coordinación de práctica, pedagógica y didáctica de la Facultad de Educación Física, la propuesta inicial que pondría en acción la integración de la investigación con la docencia, a través de la construcción, implementación y desarrollo de un proyecto de investigación formativa ${ }^{4}$.

Con la aceptación de la propuesta se asignan 11 estudiantes a la docente Nelly Teresa Martínez, gestora de la idea, quien a través del desarrollo de los tres niveles de práctica, adelantaría progresiva y secuencialmente el proyecto, que en principio se denominó Caracterización de las prácticas educativas de los docentes licenciados en educación física de Bogotá, con la idea de vincular nuevos grupos de estudiantes y profesores, si el proyecto en su evolución lo reque-

1. Reflexiones sobre la relación investigación y formación de maestros en educación física, fueron publicadas en la revista Lúdica Pedagógica No. 5 del año 2000.

2. Durante más de 15 años he tenido la experiencia de orientar práctica pedagógica, e investigación, en la licenciatura en Educación Física de la UPN.

3. Se denomina práctica inicial del maestro a los procesos de formación que le brindan al futuro licenciado, espacios para el desarrollo de competencias pedagógicas y didácticas.

4. La investigación formativa en la UPN, especialmente en la Facultad de Educación Física, surge con la intención de crear un espacio para los proyectos de que pretenden constituirse como un medio de formación profesional en lo investigativo y pedagógico, tanto de maestros, como de estudiantes. 
ría, y en la perspectiva de que se orientaría a identificar, describir y reflexionar sobre las prácticas de los licenciados en educación física egresados de la UPN. Como núcleo problémico desde el que se desarrollaría no sólo un proyecto de investigación, sino la constitución de un proceso de formación para los tres niveles de práctica pedagógica.

\subsection{El diseño del proyecto}

El diseño del proyecto se inició con la propuesta, prevista en el programa de la práctica pedagógica y didáctica I, que incluye entre sus propósitos, el construir un proyecto de investigación educativa, y la formación personal, pedagógica e investigativa de los estudiantes.

A partir del programa planteado, y de los esbozos iniciales del proyecto, se comienza un proceso de reflexión y cuestionamiento sobre lo que ha venido siendo la práctica educativa de los profesionales en el área, al mismo tiempo que se van cuestionando las prácticas de formación en las que se ha involucrado el grupo de estudiantes durante el desarrollo de la carrera.

Simultáneamente con ese análisis empírico de la experiencia, se va viendo la necesidad de lograr una clarificación conceptual sobre lo que puede entenderse como práctica pedagógica y didáctica en educación física. Esto lleva indudablemente a la búsqueda de fuentes bibliográficas y a su contrastación frente a los conceptos o ideas existentes y, en consecuencia, al descubrimiento de que las prácticas de formación han tenido básicamente una orientación de carácter tecnicista. A partir de estas reflexiones el grupo entra a problematizar las prácticas de enseñanza de la educación física a nivel escolar, analizando articulando y confrontando tales reflexiones con el estado actual de las teorías sobre la formación de maestros, práctica educativa y modelos pedagógicos.

Dado que el proyecto debería tener un sentido investigativo formal, siguiendo las exigencias del CIUP (Centro de Investigación de la Universidad Pedagógica Nacional) para sus convocatorias anua- les, los estudiantes, con la asesoría de la profesora, entran a redimensionar el concepto de investigación en un doble sentido: como práctica reflexiva y transformadora del quehacer diario del maestro en la cotidianidad de la institución educativa, y como proceso metodológico riguroso de la ciencia, tendiente a obtener conocimientos válidos. En consecuencia se incursiona nuevamente en la búsqueda de teorías y en la elaboración de conceptos.

A medida que el trabajo de discusión y clarificación conceptual se fue presentando, el grupo logró comprender las implicaciones de hacer investigación formal. Estas experiencias se van plasmando en un documento. En esta dinámica el proceso evidencia de una parte las falencias conceptuales de los estudiantes en relación con la formación pedagógica, didáctica e investigativa, y de otra, las dificultades del trabajo en grupo, ya que los niveles de apropiación y compromiso de los estudiantes mostró diferencias significativas. En el trabajo individual se vieron mejores resultados. Para la orientadora del proyecto, las cosas resultaron bastante complejas. Se requería mucha iniciativa, creatividad y visión amplia para todas las actividades necesarias en el proceso, pues aunque se trataba de un solo proyecto, no siempre se trabajaba sobre un mismo aspecto; se necesitaban, también, tareas personales para ser socializadas, de manera que todos tuvieran una visión del conjunto. En esencia la orientación del proceso favorecía el desarrollo de la visión global, tanto de la investigación en sí, como del proceso de formación, que por el tema, la Práctica Educativa del Maestro de Educación Física, se articulan.

Cuando el proyecto avanzó metodológicamente en forma global, surgió un interrogante devastador: ¿Podía la práctica que se estaba baciendo alrededor del proyecto, considerarse una práctica educativa propicia para brindar la formación pedagógica y didáctica exigida en el desempeño laboral del maestro de Educación Física? Para los estudiantes la respuesta fue positiva, para la orientadora, no. Quedó la duda de que tal experiencia, que había sido vivida dentro de un aula de la Universidad, sin trabajo de campo real, fuera la 
mejor forma de contribuir a la reflexión sobre la práctica. La pregunta debió esperar al segundo semestre para implementar una observación participante, para la toma de conciencia de lo que implica ser profesor de educación física en una institución. Se presentaron otras dificultades frente al sentido del problema a investigar, pero fueron superadas $y$ hoy el proyecto muestra los avances que a continuación se relacionan.

\section{Contenidos del proyecto}

\subsection{Problema}

En la Universidad Pedagógica Nacional, la Facultad de Educación Física viene orientando la formación de maestros, desde planteamientos que apuntan al desatrollo integral tanto de sus futuros licenciados, como al de los estudiantes que ellos orientarán en cl sistema escolar. A pesar de que los diseños curriculares en los cuales se apoya la formación de los futuros licenciados, presentan estructuras y objetivos conducentes a tal propósito en su desarrollo y a través de las asignaturas, particularmente en lo relacionado con las prácticas iniciales se observa una orientación curricular de tipo técnico. Lo que esto evidencia es que no existe una discusión académica de profesores y estudiantes al interior de la Facultad, que clarifique la diferencia entre un modelo de formación de racionalidad técnica $^{5}$ y uno de racionalidad práctica. Así mismo parece existir un desconocimiento o dificultad sobre como articular lo técnico a lo práctico en la formación.

Lo anterior conduce a que en el desarrollo de los currículos, tanto del 84 , como de los anteriores, no se encuentren procesos metodológicos y didácticos que permitan integrar estas dimensiones, enfoques o pensamientos, y de esta forma, en el desempeño profesional se convierte en prácticas de enseñanza en las que se repiten o reproducen aquellos procesos que se aprendieron como modelo; esto es, básicamente "saber como", reforzándose esta idea por el imaginario social sobre la clase de Educación Física, que la concibe como la simple mecánica de "hacer actividad física". En este imaginario no es posible suponer, y menos aceptar que esta área está hecha también para pensar: el por qué o el para qué, del movimiento humano.

Afirmaciones como que la clase de educación física no aporta o contribuye a la formación integral del individuo, porque solo se centra en la adquisición de habilidades netamente deportivistas; $\mathrm{o}$ que la clase es espacio para recreos supervisados, o que el maestro se dedica a entregar un balón, para que los niños hagan lo que quieran, sin una orientación pedagógica por parte del docente, dejan ver que en la comunidad tanto académica como de padres, existe un pensamiento, una creencia, o una imagen sobre el deber ser de la clase, y que trasciende el tecnicismo motor.

El contexto anterior dirigió la mirada a saber cuáles son las intencionalidades que mueven a los maestros en el desarrollo de sus clases, es decir, ¿qué pensamientos y creencias tiene el licenciado en educación fisica que labora en la básica secundaria sobre el sentido de su clase? ¿Trasciende en forma consciente el interés técnico de sus actividades?

El primer grupo de estudiantes avanza hasta la formulación de la primera parte de la pregunta: ¿qué pensamientos y creencias orientan a los maestros de educación física en la realización de sus clases? El grupo pensaba que logrando una descripción sobre la clase podría derivar los sentidos de cómo se desarrolla. Sin embargo, es el segundo grupo de estudiantes en su nivel de práctica II, que descubre, después de una entrevista informal dentro de la UPN, que la orientación técnicista e integral de

5. Se concibe como racionalidad o interés técnico, al pensamiento que orienta la actividad humana, como un trabajo de fabricación o producción, según modelo de acuerdo con el cual se construye un objeto. Al realizarse este tipo de actividad o trabajo, aparece "un producto"; cuando la idea (eidos) se transforma en realización de la acción (poietiké) a través de la disposición humana de la técnica (tekné). En este sentido, la forma de actividad humana puede asumirse como un oficio. Esta forma de actividad difiere según Arendt, Aristóteles y Kemis, de la "acción" (en griego, praxis), la que se sitúa como "práctica" y que tiene lugar, entre dos personas, a través de la interacción (el habla) y que implica juicio y deliberación democrática, y preocupación por el bien común y no la simple ejecución de la idea o modelo.

6. El primer grupo de estudiantes se avanzó hasta la formulación general de instrumentos. El segundo asume el documento y entra en apropiación de su sentido y coherencia. 
la clase requerían ser conceptualizadas por el grupo investigador, para poder ser observadas y reconocidas en el trabajo de campo.

A partir de este momento la pregunta de investigación es verdaderamente asimilada por profesores y estudiantes, y empieza para el proyecto realmente la fase de comprensión y apropiación. Las preguntas analíticas, por su parte, se resignifican y se concretan, quedando formuladas así:

- ¿Cuál es el perfil profesional del licenciado en educación física que actualmente orienta la clase?

- ¿Qué finalidades persigue el maestro de educación física en el desarrollo de la clase?

- ¿Qué relación establece el maestro de educación física entre los contenidos y actividades que desarrolla en la clase, y las finalidades que le asigna a la educación física?

- ¿Cómo concibe el maestro de educación física el desarrollo de los estudiantes?

- ¿En qué consisten los procesos metodológicos y las técnicas que usa el maestro de educación física en la clase?

- ¿Desde qué concepciones maneja el maestro de educación física las relaciones de poder en la clase?

A partir de estas preguntas se plantearon los siguientes objetivos para el proyecto

\subsection{Objetivos}

\section{General}

- Conocer y comprender, desde la perspectiva de la educación técnica y la educación integral, la forma como orientan la clase los licenciados en educación física que laboran en la básica secundaria de Bogotá.

\section{Específicos}

- Describir e interpretar los pensamientos y creencias que tiene el licenciado en educación física en el desarrollo de sus clases, en relación con las finalidades, contenidos y actividades, concepciones de desarrollo, procesos metodológicos y relaciones de poder.
- Contrastar tales pensamientos y creencias con las realizaciones concretas que le posibilita el contexto real de su desempeño.

- Elaborar una propuesta pedagógica que, teniendo en cuenta los pensamientos y creencias del maestro sobre la educación física y la realidad del contexto de desempeño, aporte a la reflexión y transformación de la clase.

\subsection{Metodología}

\subsubsection{Fundamentos}

Este proyecto parte de la afirmación de que las prácticas educativas del maestro de Educación Física pueden ser conocidas ya que su existencia no sólo puede ser verificada por su presencia institucional sino porque ellas son significativamente expresadas a través de lenguajes verbales y corporales, y por infinidad de acciones mediante las cuales el maestro da a conocer parte de su pensamiento e intencionalidad educativa; tales intencionalidades se soportan en saberes, creencias, conocimiento y valoraciones que se han constituido en los diferentes espacios o contextos de la historia del maestro, como son: la familia, la escuela, el barrio, la formación universitaria, la experiencia laboral y el acercamiento a los medios masivos de comunicación, entre otros. Todo ello puede confluir en el pensamiento y acción del profesor, haciendo de sus prácticas un campo heterogéneo y complejo de estudiar o conocer. Aunque las prácticas educativas del maestro pueden presentarse en distintos momentos y espacios de su quehacer profesional, las que en este proyecto se estudian, se circunscriben únicamente a la "clase de educación física", denominada así, o por lo menos con esa intencionalidad.

Ahora bien, estas prácticas educativas pueden estar soportadas además en algún tipo de teorías que sirven para orientar y explicar la acción del maestro durante su realización. Se puede afirmar que el profesor de la clase tiene una forma particular de entendimiento de lo que hace y que no es muy evidente; así que sus comportamientos se muestran a la vista de un observador corriente, como pragmatismos carentes de sentido. Sin em- 
bargo, cuando el docente es interrogado sobre los soportes de su quehacer, parece tener una explicación para ello. En consecuencia, el modo particular de conocer este tipo de prácticas, se muestra asequible al método etnográfico, por cuanto busca hacer una caracterización de la dimensión cultural de las prácticas educativas de los maestros de educación física tal como ellos las perciben, esto es, como las comprenden y como las explican desde su cotidianidad. Esta caracterización será descriptiva - interpretativa, para lo cual se tendrá en cuenta una primera categorización de la información surgida de los textos verbales enunciados por los maestros. A partir de ello se hará una confrontación frente a los marcos teóricos y conceptuales planteados como soportes para el proyecto.

Las técnicas para la recolección de la información serán: el cuestionario, la entrevista y la observación, con las cuales se esperan confrontar las diversas informaciones y dar más validez al conocimiento que se obtenga.

\subsubsection{Etapas del proceso}

El proyecto está pensado para ser realizado en dos etapas, una primera que comprende tres fases: fundamentación conceptual e investigativa; comprensión y apropiación; y aproximación al conocimiento de las prácticas; $y$ una segunda etapa encaminada a generar conocimiento analítico sobre la práctica, desde el cual poder sustentar una propuesta para su reflexión y transformación.

\subsubsection{Población y muestra}

La población objeto de estudio estuvo pensada inicialmente por el total de los maestros licenciados en educación física que realizan la clase en los 2.853 colegios oficiales y privados de Bogotá, distribuidos en las 20 zonas que conforman el perímetro de la ciudad. Finalmente se decidió tomar una muestra conformada por el $10 \%$ del total de colegios, que cuenten con profesor de Educación Física, en el nivel de básica secundaria.

\subsubsection{Delimitación y alcances}

Teniendo en cuenta que este proyecto busca ante todo constituirse como un medio para la formación pedagógica e investigativa de los estudiantes de la licenciatura en educación física, antes que obtener resultados en términos de la investigación misma y de su validez y confiabilidad. Además se prevén algunas limitaciones como las siguientes:

Selectividad de la muestra: El proyecto no cuenta con los estudiantes y profesores suficientes para una cobertura de la magnitud pensada (el grupo actual es de ocho estudiantes y tendrá dos semestres más de práctica); el hecho de que la práctica pedagógica y didáctica, como asignatura del plan de estudios de 1984, ya no está vigente, por la implementación del nuevo proyecto curricular del año 2000 , la muestra planeada se reduce a la selección de un grupo de profesores, máximo 50, que llene la característica de representatividad y que cumplan como mínimo, las siguientes condiciones:

- Ser licenciados en educación física de la UPN.

- Poseer experiencia profesional, de mínimo cinco años, como profesor de educación física en la básica secundaria.

- Estar dispuesto a responder el cuestionario.

- Permitir la observación de una clase y una entrevista dialogada

\subsubsection{Diseño de instrumentos}

La técnica empleada en el proyecto fue la de cuestionario. Su diseño partió de la consideración de las cinco preguntas básicas de la pedagogía o "criterios de elegibilidad para la pedagogía" (Flórez Ochoa, 1997) que son procesados en cinco tópicos fundamentales que enmarca los modelos pedagógicos a saber: las metas, los contenidos, procesos de desarrollo, el método y las relaciones interactivas maestro - estudiante, contextualizados desde unos frentes que den cuenta del perfil profesional del licenciado. El cuestionario en esencia, consta de 7 tópicos a saber: perfil profesional, ideal de educación física, metas de la clase, contenidos de la clase, metodología, procesos de desarrollo y relaciones maestro - estudiante, elaborado con preguntas abiertas y cerradas. Para la complementación de su diseño se hicieron tres entrevistas a profundidad 
sobre estos tópicos y con base en las respuestas precisaron las preguntas del cuestionario.

\subsubsection{Marco referencial}

\section{- Estado del arte}

La aproximación muestra que un número importante de investigadores, dentro y fuera de Colombia, se han dedicado al estudio de lo que sucede con las prácticas de enseñanza de la Educación Física de los maestros en las escuelas. La investigación, Características del proceso de práctica en la formación de futuros docentes de educación física, realizada en los centros superiores de formación del profesorado, en Andalucía, España, muestra que "las prácticas por sí mismas, y tal como se desarrollan habitualmente, no solo sirven como espacio de experimentación, sino como instancias de reproducción, induciendo un modo de concebir la escuela y la enseñanza, en detrimento de otros modos y concepciones alternativas. La inmersión acrítica del futuro docente en el clima profesional de la escuela, potencia el desarrollo y la proliferación de prácticas uniformes, rutinarias y estereotipadas, exigidas de alguna manera para la permanencia y reproducción del estatus quo" (Candau, 1997); como se infiere, los resultados de este estudio evidencian una problemática similar a la planteada en este proyecto, al mostrar que las prácticas de los licenciados en Educación Física se convierten en espacios de reproducción de formas instrumentales y no de reflexión. Sin embargo, otro estudio publicado en el artículo: "La entrevista rememorada en la formación inicial de los docentes de Educación Física", afirma que "la formación inicial de los enseñantes está evolucionando y progresando de un modelo de traspaso de conocimientos (racional - técnico) a otro basado en el desarrollado por competencias (reflexión - práctica)", (Camerino, 1997). $\mathrm{El}$ autor destaca que cuando se utilizan durante la formación, estrategias, como la entrevista, sobre imágenes en video, de episodios que los estudiantes han realizado en sus prácticas, se llega a análisis complementarios sobre la propia actuación, lo que conduce o facilita la toma de conciencia de la complejidad y una mejor comprensión de la acción educativa. De esto se concluye que una formación investigativa como la que se ha generado con este proyecto, de reflexión sobre la propia práctica, contribuye positivamente a la formación del futuro licenciado.

Finalmente, cabe destacar que en Chile, la Universidad Metropolitana de Ciencias de la Educación, en uno de sus proyectos innovadores, ha tomado la decisión de crear nuevas metodologías para realizar transformaciones en la formación de profesores en el plan FID ${ }^{7}$, con el propósito de iniciar desde los primeros semestres el contacto de los estudiantes con la escuela.

Asimismo, en el proyecto de investigación Los imaginarios de los adolescentes ante la clase de Educación Física ${ }^{8}$, se pone de relieve las actitudes mostradas por los adolescentes en torno a la clase de educación fisica, en las que se puede evidenciar cierta apatía, pero a la vez agrado por practicar actividades físicas no escolarizadas. Aunque el estudio no se ha terminado, la problemática esbozada pone alerta e invita a reflexionar sobre lo que está pasando con la clase de Educación Física, finalidad que en parte tiene este estudio al intentar descubrir las intensionalidades de la clase, desde la visión del maestro.

\section{Aspectos teórico-conceptuales}

La elaboración de este aspecto del proyecto ha sido uno de los más difíciles, en la medida en que el grupo de estudiantes al entrar a cursar la práctica I, evidenció muchos vacíos conceptuales, no sólo en cuanto a la concepción de investigación, sus enfoques y metodologías, sino de los temas básicos de la formación pedagógica, que requieran ser clarificados desde el problema de investigación, como son: educación, educación física, práctica educativa, pensamientos y creencias y la clase de educación física, entre otros.

7. FID, Formación Inicial Docente

8. MURCIA, Napoleón et al. "Los imaginarios de los adolescentes ante la clase de Educación Fisica", Investigación Institucional de nivel nacional, en proceso. 2000 . inédito. 
Hasta el momento se tienen algunas claridades conceptuales que han permitido la reformulación del problema, de los objetivos y de la metodología. En esencia, el proyecto aborda la educación como tema central y la concibe como un proceso dinámico que se cumple por etapas a través de toda la vida del individuo, con la finalidad de buscar permanentemente el desarrollo humano, dentro de un contexto social que le permita adquirir y renovar los valores significativos para su cultura. Así, la educación debe entenderse y asumirse como una actividad social que tiene dos funciones fundamentales: mantener la producción científica, conceptual, técnica y artística que ha elaborado la humanidad y otra que debe perseguir siempre la formación del sujeto; es decir, el ascenso cada vez mayor a "ser humano". En esencia, la educación debe entenderse como todo un sistema de procesos, interacciones y prácticas individuales, sociales, culturales, formales, informales y no formales, que constituyen un contexto multidimensional, con intencionalidades expresas o tácitas de influir en la constitución del ser humano. Pese a la complejidad de factores que intervienen en la educación del ser humano, es deseable que se busque el desenvolvimiento pleno de las potencialidades humanas en sus dimensiones del pensar, sentir y actuar. En otras palabras, la educación debe apuntar a la integralidad humana, soportada sobre cuatro pilares básicos, como lo plantea la UNESCO: "Aprender a conocer, aprender a hacer, aprender a vivir juntos y aprender a ser" (UNESCO, 1996: 95-96).

Dentro de estos procesos educativos se inscriben de manera expresa, los conducentes a la formación de maestros, lo que debe apuntar a crear en los educadores un modo de percibir en el que se integren el conocimiento y el pensamiento, para manifestarse armoniosamente sobre la sensibilidad y el carácter. La formación no es sólo resultado espiritual sino un proceso interior que abarca mucho más que el cultivo de aptitudes y talentos humanos; el ejercicio de estos son solo un medio. La formación incluye o apunta a la aproximación por entero y perdurablemente, de aquello y por lo cual uno se forma; es como el saber moral que nunca se olvida.
Así las cosas, la formación de educadores implica una gran responsabilidad para las facultades de educación, y en este caso, para las de educación física, particularmente de la Universidad Pedagógica $\mathrm{Na}$ cional, por ser la encargada oficialmente de tal mención. Esto desde luego hace parte de la preocupación que impulsa este estudio, ya que en el fondo cabe preguntarse: ¿Cómo ha sido la formación de los licenciados en educación física?, ¿qué de ella se evidencia en sus prácticas de clase?

Como se enunció anteriormente, en la educación intervienen diversos procesos, uno de ellos está relacionado con la educación física, la que desde luego ejerce una influencia importante en la formación global del sujeto, razón por la cual ha sido pensada no sólo como necesaria, sino indispensable, hasta llegar a concebirse como educación integral en sí misma, al apuntar al desarrollo del individuo en sus dimensiones: física, psicológica, afectiva, cognoscitiva, estética y ética, como lo expresara José María Cagigal, quien considera a la educación física como el más básico modo natural de educar al niño de una manera globalizada. Planteamiento que a pesar de haberse escrito en 1979, sigue vigente y es retomado en los textos modernos como Deporte y Ciencia de Juan Rodríguez López (1996), con la intención de demostrar que la educación física no debe reforzar el intelectualismo y la tecnificación, revindicando que la tarea de la educación física es: "descubrirse y conocerse a sí mismo, aceptar sus propias realidades, descubrir el entorno social y comunicarse con él...” (Rodríguez, 1996). En consecuencia, se considera en este proyecto, que la educación física es "la educación a través de su cuerpo, el cual es la primera instancia que permanece y acompaña al hombre durante toda su vida... Con el ejercicio integral de sí mismo, el hombre se ejercita para capacitarse en todos sus niveles, desde la conducta superior hasta las destrezas mecánicas y capacidades sicológicas. Se capacita en el hábito al esfuerzo, en la formación del carácter, en el principio de respeto a sí mismo (a su cuerpo) y a los demás (en el contacto que con los demás tiene por medio de la conducta corporal), en la instauración de una moral que arranque desde el pacto del hom- 
bre consigo mismo, con sus propias emergencias corporales" (Cagigal, 1977: 84-85). El autor hace una severa crítica en el sentido de afirmar el desconocimiento de lo que puede ser la educación física, incluso entre los profesionales del área. Se ha llegado a que se gradúen educadores que no están formados para hacer de la educación física, la forma más natural, sencilla, cómoda y alegre de educar integralmente al niño. Cabe preguntarse si la crítica hecha por el pensador clásico de la educación física hace 38 años, sigue vigente, puesto que aunque la formación de educadores ha intentado mantener la intencionalidad de la integralidad, en la práctica de la escuela parece no observarse.

Sin embargo, no puede afirmarse rotundamente que el profesor de educación física no tenga una intencionalidad formativa en lo bumano, a través de las actividades técnicas que ejerce. En este sentido, el proyecto pretende identificar cuáles son los pensamientos y creencias que soportan las actividades en la clase, así como el espacio central y fundamental de la acción educativa del maestro de educación fisica, ya que este proyecto concibe la clase de educación física como el espacio básico de la práctica educativa, a través de la cual se ponen en evidencia e interacción los conocimientos, creencias y habilidades comunicativas; es decir, todo lo que constituye el pensamiento alrededor de la educación, y de la educación física en particular, es lo que permite afirmar que la clase es el elemento primigenio de la práctica educativa del licenciado.

Las clases se conciben como las experiencias planificadas, previstas, mínimo en su globalidad, que buscan poner en relación las teorías, las concepciones o las ideas, con la acción, generando esquemas prácticos para la función del profesor. "El ámbito de la práctica profesional es el espacio de la acción y la comprensión intersubjetivas, sus señas de identificación son la imprevisibilidad y la incertidumbre" como lo plantea Carr (1998: 75). El sentido de imprevisibilidad e incertidumbre implica que el educador nunca puede estar completamente seguro de que todas las actividades realizadas durante una clase constituyan una experiencia significativa y rica para los niños, pese a que las haya previsto o pensado con antelación. Sin embargo, no es así como la ideología de nuestra época afronta la imprevisibilidad de la praxis. "En una época en que la subjetividad y la imprevisibilidad son inaceptables, y que por el contrario prima la certeza, o por lo menos, niveles elevados de probabilidad, si la práctica no puede proponernos la certeza que se le reclama, entonces, la acción del momento debe reducirse a trabajo, el juicio a la técnica y la deliberación al cumplimiento de reglas." (Carr, 1998: 76). Desde esta perspectiva, es como se ve hoy al maestro de educación fisica, y tal vez, también él se asuma, y en consecuencia, en vez de considerar la educación como un bien humano, se piensa y describe el proceso educativo como un sistema que genera productos a los que hay que medir para determinar su calidad; y lo que es más grave, se piense y se crea que es la enseñanza la aplicación, únicamente, de técnicas.

Esta tecnificación de la práctica no sólo es evidente en la producción de conocimientos. Cuando se separan los resultados de la investigación de la acción realizada en los contextos. La aplicación tecnológica concede mucha importancia al resultado de la acción, es decir, a lo que produce; sin embargo, la práctica profesional (praxis) tiende a valorar la acción en sí misma, por encima de los resultados, o sea, considera más importante actuar prudentemente, que cumplir meticulosamente unas reglas para corregir unos resultados.

En principio, el sentido de las prácticas pueden presentarse en dos direcciones: orientadas por un pensamiento o juicio práctico, o por un pensamiento o juicio técnico. La primera, corresponde al tipo de acción humana que tiene como propósito la interacción humana; es decir, la relación humana basada en el diálogo, está dirigida a la consecución de un fin que no es la producción de un artefacto o cosa, sino la realización de algún bien moralmente conveniente; la práctica no es en sí misma neutral. El bien para cuya consecución se hace la práctica, no puede elaborarse, sólo puede hacerse. Funcionalmente, la práctica no puede entenderse 
como un tipo de maestría técnica para alcanzar algún fin relacionado con ella... Así, la praxis es diferente de la poesis (o práctica técnica), que implica un trabajo concreto de fabricación que se lleva a cabo según un modelo. Así las cosas, la práctica del profesor de Educación Física puede verse o darse en cualquiera de estos dos sentidos, y es precisamente la dimensión técnica, enfoque cuestionable, la que constituye el centro de interés de este estudio.

\section{Algunos resultados y aportes}

En sus cinco semestres de desarrollo, el proyecto permite mostrar algunos avances, que se clasifican en dos: de investigación y de formación.

\section{Investigación}

$\mathrm{Al}$ analizar los datos del instrumento piloto (cuestionario, con preguntas cerradas y abiertas, para explicar o argumentar las respuestas, aplicado a 30 licenciados en educación física que laboran en Bogotá, en torno a la formación académica, experiencia profesional, producción intelectual, perfil educativo familiar, actividad cultural y expectativas sobre' la carrera y desempeño en la clase), se pudo establecer que: la formación predominante de pregrado es bachillerato clásico, que el 100\% de los encuestados son licenciados en Educación Física, $86.6 \%$ de la Universidad Pedagógica Nacional, 6.6\% de la Tecnológica de Tunja, y 6.6\% de la Universidad de Cundinamarca. El 16\% obtuvo el título entre 1.976 y 1.978 , un $46 \%$ entre 1.980 y 1.989 , mientras que el $30 \%$ se graduaron entre 1.995 y 1.998 . En su mayoría, el 73\%, de los profesores, son hombres. Cerca al $63 \%$ de los encuestados lleva laborando un promedio de 16 años; lo que puede hacer pensar que existan deferencias en los pensamientos y creencias en relación a la clase de educación física, entre los antiguos y los graduados después de 1995.

Con respecto a la preparación académica se observa que el $23 \%$ de los encuestados no ha hecho especializaciones, y el $80 \%$ ninguna maestría. El $33 \%$ se ha especializado en edumática y adminis- tración deportiva, y el $23 \%$ en pedagogía y didáctica de la educación física, y entrenamiento deportivo. Es significativo mostrar que en educación informal, como congresos, diplomados y seminarios, se promedian dos por cada profesor (en total 66), la mayoría relacionados con educación física y algunos deportes como voleibol, natación, fútbol, gimnasia, y patinaje. Por lo expuesto, se puede afirmar que existe, un mediano interés por la actualización y profundización.

En lo relacionado al factor empleo, el $76 \%$ labora a nivel de la básica secundaria y un $23 \%$ en primaria y secundaria a la vez; los porcentajes coinciden para el sector oficial y el público. Aproximadamente el $53 \%$ de los maestros trabajan en colegios de estratos tres y cuatro, y el $34 \%$ en estrato uno y dos, solo el $16 \%$ lo hace en estrato cinco y seis.

En cuanto a producción académica, del $60 \%$ de los maestros que considera haber realizado algo (32 del total de la muestra), solo el $18 \%$ ha publicado en revistas o a través de libros. El $46 \%$ manifestó no haber realizado ningún tipo de producción. En general, se puede concluir, de una parte, que no hay motivación en el grupo de profesores por la construcción conceptual en el área, y de otra, que la que existente no se da a conocer; es decir, la profesión muestra una gran falencia en el avance investigativo.

En lo referente a las actividades culturales que frecuenta el grupo indagado, el deporte y las actividades físicas, ocupan el primer lugar; en segundo lugar, la lectura. De igual manera, la prioridad de participación en sus colegios son los juegos y torneos, las jornadas culturales y deportivas; muestra de la fuerte inclinación por la actividad física y el rendimiento en el área en que se formaron profesionalmente. El 56\% escogió la carrera por gusto al deporte. Vale la pena, entonces, preguntarse si tal preferencia sigue marcando la orientación de la clase.

\footnotetext{
9. El cuestionario diseñado por el grupo surgió de la comprensión de los elementos conceptuales que pueden tenerse en cuenta en un modelo pedagógico, desde el punto de vista teórico. Así mismo se aplicó una entrevista a profesores de educación física para precisar mejor las preguntas a plantear en cada aspecto del modelo.
} 
Es cuestionante encontrar que las preferencias que motivaron el estudio de la carrera de educación física haya sido: el ejercicio físico, la práctica deportiva y el rendimiento $(63 \%$ de los encuestados); un 13\% pensó la carrera para el ejercicio docente; y el 13\% para el desarrollo integral humano. El 10\% desconocía lo que iba a estudiar. $\mathrm{Al}$ preguntarles si la formación recibida en la Universidad modificó su idea previa sobre la educación física, el 36.6\% afirmó que su idea inicial cambió considerablemente, el $20 \%$ sólo en cuanto a la finalidad pedagógica y educativa.

En relación con la clase, el 100\% considera que cambió el modelo didáctico dado por la Universidad, por que la realidad es diferente: $56.6 \%$ plantea que la experiencia sugiere otros modelos, el $16 \%$ optó por la formación integral y no deportiva, y otro $16 \%$ por otros postulados. También se encontró que el $33 \%$ concibe la clase como un espacio para la formación en valores, hábitos y relaciones interpersonales a través del movimiento; finalidades correspondientes más a los planes de estudio de los años 60 al 80 . El 26\% la consideró como espacio para la formación integral, al afirmar que desarrolla aspectos cognitivos, psicológicos, volitivos y físicos.

Con respecto a la finalidad que debe cumplir la clase, el $21.3 \%$ piensa que debe ser para la formación de la persona y el $17.7 \%$ para desarrollo motor. También fueron planteadas con porcentajes del $10 \%$, finalidades como: hacer actividad física, pasarla bien, ayudar a la socialización y reflexionar.

\section{Formación}

$\mathrm{Al}$ revisar los resultados de la información obtenida a través del cuestionario aplicado por el primer grupo, y confrontarla con la problemática planteada, se detectó que la forma como estaban orientadas las preguntas no permitían obtener una respuesta consecuente. Esta situación ya se había presentado con el primer grupo, semanas antes de probar el instrumento; la formulación de la pregunta de investigación no se derivaba lógicamente de los hechos que soportaban la situación descriptiva del problema. En consecuencia se siguió un proceso didáctico que condujo a su reformulación; por ello considero que el mayor aporte a la formación del equipo ha sido el mejoramiento de la capacidad de análisis y de construcción de pensamiento lógico.

Desde el punto de vista de la formación investigadora, los dos grupos que han participado en el proyecto, consideran haber desarrollado una actitud crítica frente a lo que implica ser profesor de educación física, concretamente frente al espacio de la clase. El proceso de observación condujo al redimensionamiento de las finalidades que se pueden asignar a la educación física, más allá del tecnicismo; empezando a construir un pensamiento pedagógico con referentes que apuntan a la integralidad del proceso educativo. También es importante destacar el aprendizaje obtenido, tanto en la estructuración y fundamentación conceptual de un proyecto de investigación educativa, como en la adquisición de habilidades comunicativas para la exposición de ideas y argumentaciones, no solo en el plano oral, sino fundamentalmente en la escritura de textos. El aspecto didáctico, si bien no fue relevante para el primer grupo, si lo ha sido para el segundo, en la medida en que la apropiación del proyecto se hizo no solo a partir de la lectura y comprensión del documento elaborado por el grupo, sino de la implementación y desarrollo de clases de educación física en la básica secundaria, tratando de darle sentido a lo que pretende la pregunta y los objetivos del proyecto.

Para finalizar este artículo, quiero destacar lo que para mí como orientadora ha significado la construcción y desarrollo del proyecto. Indudablemente el aporte se ubica en la comprensión de lo que implica que los procesos de investigación articulen el desarrollo curricular, lo que en este proyecto se ha posibilitado, gracias a que el objeto de estudio ha permitido la profundización en los campos de formación pedagógico y didáctico, disciplinario o específico y el científico hermenéutico, denominado de Integración, en el currículo de 1984. 


\section{Bibliografía}

CAGIGAL, José María. (1977). Cultura intelectual y cultura fisica. Buenos Aires, Argentina. Kapeluz. p. 84-85.

CAMERINO, Foguet; OLEEGUER y PRADAS. (1997). La Revista Electrónica Interuniversitaria de Formación de Profesorado. Instituto Nacional de Educación Física, Departamento de Didáctica de la Universidad de Barcelona, España.

CANDAU LLERA, María. (1989). La didáctica en cuestión. Madrid-España.

CARR, Wilfred. (1998). Calidad de la enseñanza e investigación-acción. Sevilla, España. Diada Editora.
FLOREZ OCHOA, Rafael. (1997). Hacia una pedagogia del conocimiento, Bogotá. Ed. Mc Graw Hill.

RODRÍGUEZ LÓPEZ, Juan. (1996). El racismo. Citado por CAGIGAL, José María (1977). Cultura Intelectual y cultura física. Buenos Aires. Argentina. Kapeluz. p. 34.

UNESCO. (1996). La Educación un tesoro. Informe a la UNESCO, presentado por la comisión internacional sobre educación para el siglo XXI, Madrid. Santillana. 